\title{
The importance of gender in health problems
}

\author{
TOINE LAGRO-JANSSEN, SYLVIE LO FO WONG \& MARIA VAN DEN \\ MUIJSENBERGH \\ Department of General Practice, Radboud University Nijmegen Medical Centre, Nijmegen, the Netherlands
}

\begin{abstract}
Objective: To investigate gender differences in health problems in general practice. Methods: We performed a study using data from the Continuous Morbidity Registration (CMR) Nijmegen. To study the distribution by gender we analysed overall morbidity per 1000 patients years over a period of 10 years. Secondly we calculated the influence of gender by diagnostic rubric. For each diagnosis in the rubric of musculoskeletal disorders, we calculated incidence and sex ratio related to socio econonomic status (SES). We did the same concerning the referral rates for cardiovascular disease (CVD). Results: Women had significantly more health problems than men, most striking in the age group 25-44 years, due to screening and reproductive health problems. Concerning gender differences related to SES in muskulo skeletal disorders we found the lower the SES the more prevalent is osteoarthritis of the knee in women. The sex ratios in CVD showed men as leading sufferers. Decreasing SES resulted in increasing incidence of CVD and a lower referral rate to the cardiologist in women.

Conclusion: Sex differences appear in the prevalence of health problems, risk factors and access to medical care. They also can influence the course of diseases. Health problems in men and women also vary according to socio-economic status, meaning that gender is strongly intertwined as risk factor with socio-economic status, ethnicity, and age.
\end{abstract}

Key words: Gender, socio-economic status, morbidity, musculoskeletal disorders, cardiovascular diseases, IBD, referral

\section{Introduction}

Gender is an essential determinant of social outcomes, including health. Moreover, gender cannot be separated from other social identifiers such as ethnicity, age, or socio-economic status. Although the impact of ethnic diversity or socio-economic status on health can hardly be underestimated, gender does play a pivotal role within these social identifiers.

Differences in health between men and women are due to the interactions of biological, psychological, and social factors (1). Attention to gender differences is relevant not only in reproductive functioning, but also in other health fields, such as the prevalence of diseases, risk factors, presentation of health complaints, impact of illness, and the treatment and course of diseases (2). The World Health Organization's "Gender Policy" recommended that an integration of gender considerations become standard as a contribution to better health for women and men (3).

Extensive research on sex differences has shown that women have higher rates of morbidity and utilize medical care more often than men $(2,4)$. Although research has revealed strong evidence for the presence of sex differences in morbidity and medical care utilization in general, the relation between gender and specific types of morbidity and health problems is only being gradually clarified.

Sex differences in morbidity may be based on selfreported perceptions of symptoms, or on physicians' evaluations of health status through clinical diagnosis. In the present study, we analysed medical diagnoses and health problems registered by general practitioners working in the practices of the Continuous Morbidity Registration (CMR) Nijmegen project.

The aim of the study is to investigate gender differences in health problems in general practice.

Correspondence: A. L. M. Lagro-Janssen, Women's Studies in Medicine, Department of General Practice, Radboud University Nijmegen Medical Centre, 117 HAG, PO Box 9101, 6500 HB Nijmegen, the Netherlands. Tel: +31 2436131 10. Fax: +31 24354 18 62. E-mail: a.lagro-janssen@hag.umcn.nl 
We wish to analyse some specific health problems as examples to give more insight into the role gender might play in health problems in general. We therefore focus firstly on gender differences in the prevalence of overall health problems in relation to age, followed by gender differences related to socioeconomic status in musculoskeletal disorders and, more specifically, on osteoarthritis of the knee. Secondly, we study gender and socio-economic status in cardiovascular diseases and referrals to secondary specialist care. Finally, we conclude with an example of gender differences in the course of one health problem, namely inflammatory bowel diseases (IBD).

\section{Methods}

The data used were obtained from the CMR (5). Firstly, we compared overall male and female health problems, by age and by severity, as presented in primary care. Overall morbidity for patients in this study comprises the total number of registered ICHPPC-2 health problems per 1000 patient years over a period of 10 years. Secondly, we calculated the influence of gender per diagnostic rubric, by dividing the sex difference in incidence per diagnostic rubric by the sex difference in overall diagnostic rubrics, multiplied by 100 . Incidence is defined as the number of new cases per 1000 patient years, in which one patient year is defined as one patient who has been followed for 1 year. Prevalence reflects the sum of the number of newly presented health problems and ongoing health problems per 1000 patient years. For each age group, incidence and prevalence of health problems were computed.

We used four categories for severity: serious, i.e., the disease threatens the patient's life or has a longterm impact on the patient's functional capacities; moderately serious, i.e., the disease temporarily interferes with the patient's functional capacities; not serious, i.e., the disease does not influence the patient's functional capacity. A fourth category was used to indicate not otherwise classified.

For each diagnosis in the rubric of musculoskeletal disorders, we calculated incidence and sex ratio. Sex ratios represent the incidence of women divided by the incidence of men. We did the same concerning the referral rates for angina pectoris and myocardial infarction.

Morbidity per 1000 patient years is calculated with a $95 \%$ confidence interval (CI) for men and women separately. We calculated relative risks, and we defined this as the chance of women having a diagnosis as compared with men. Two-tailed Student's $t$ tests were carried out to test differences in male and female rates.
For comparison between men and women in complications in the course of IBD, we used Cox proportional hazard analysis.

In our study, we defined results with a $p$ value $<0.05$ as significant.

\section{Results}

\section{Overall morbidity}

Women had significantly more health problems than men (2286 and 1589 per 1000 patients, respectively; $p<0.001)$. For each age group, we computed the prevalence of health problems for men and women. The sex difference in the prevalence of health problems was the most striking in the age group 25 to 44 years of age $(p<0.001)$ (Figure 1$)$. Screening and health education (contraception, health advice and prevention, screening and diagnostic procedures) accounted for the major part of the sex differences, followed by genitourinary system diseases and diseases of the respiratory system (Figure 2). In all four severity categories, the incidence of presented health problems was higher for women than for men; however, per category, the percentage of overall diagnostic rubrics was smaller for women than for men in all four severity categories, except for the category "not otherwise specified" (Table I).

\section{Musculoskeletal disorders}

An evident outnumbering of women was clearly seen in all diagnoses of musculoskeletal orders, except for herniated disc (Figure 3). Osteoarthritis of the knee is twice as prevalent in women compared with men, especially among the elderly (at age $\geq 75$, the prevalence is 129.3 [95\% CI 115.3-143.3] for men versus 279.7 [95\% CI 264.1-295.3] for women). The lower the socio-economic status, the more

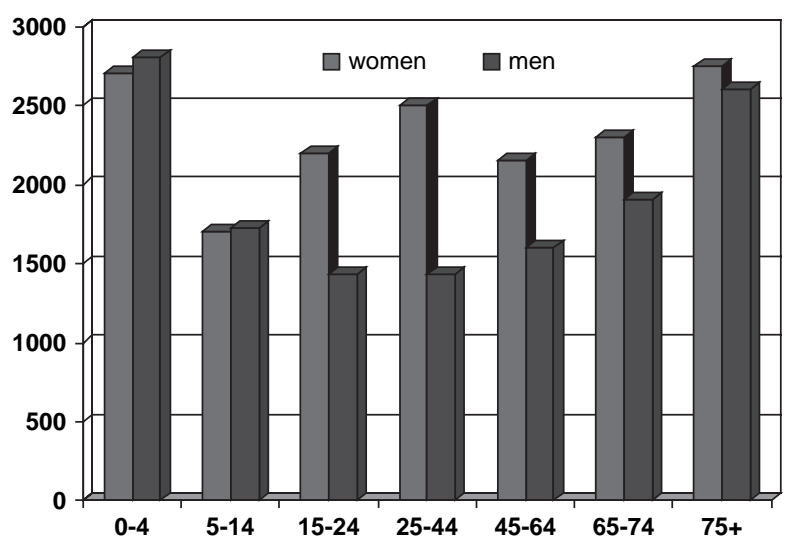

Figure 1. Overall health problems: incidence per 1000 men and 1000 women per year, by age. 


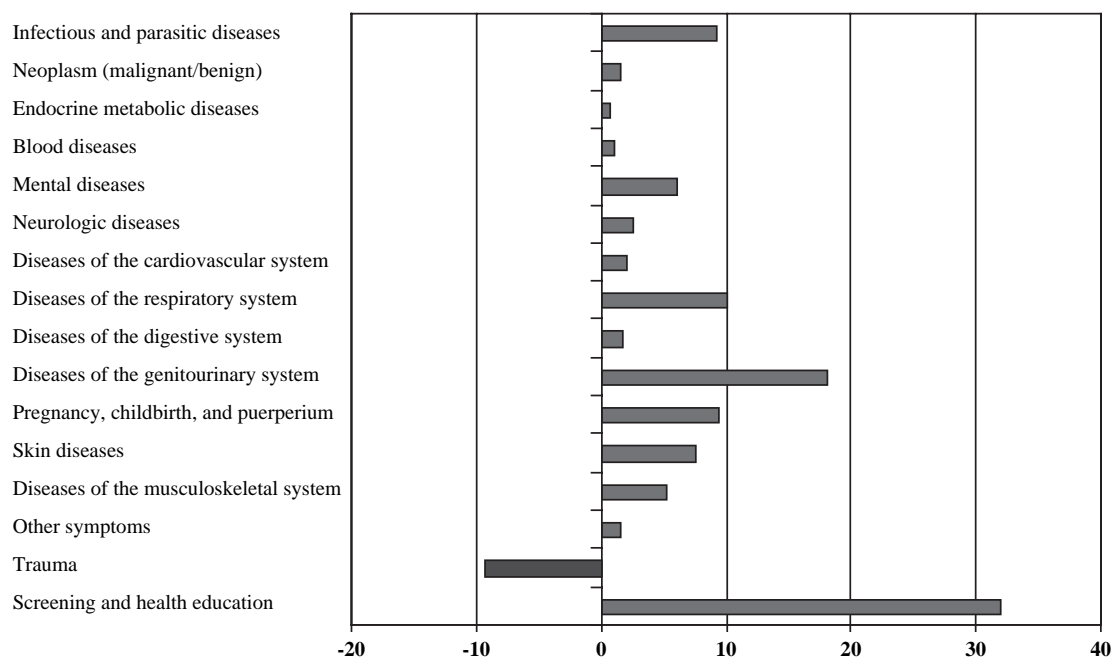

Figure 2. Contribution (\%) to sex difference in overall morbidity per diagnostic rubric.

prevalent is osteoarthritis of the knee in women. In the low and middle socio-economic classes, women present more osteoarthritis of the knee than men: the prevalence in patients of low socio-economic status is 23.7 (95\% CI $21.8-25.7$ ) in men versus 48.1 (95\% CI 45.2-50.9) in women; the prevalence in patients of middle socio-economic status is 15.5 (95\% CI 14.0-17.0) in men and 35.9 (CI 33.7-38.1) in women.

\section{Cardiovascular diseases}

The sex ratios in the incidence of myocardial infarction and angina pectoris showed a reverse outcome, with men as leading sufferers: 3.4 and 3.0 for men and 2.1 and 2.1 for women, respectively $(p<0.01)$ (6). However, the same phenomenon occurred when comparing the incidence in men and women by socio-economic status: decreasing socio-economic status resulted in an increasing incidence of cardiovascular diseases, in particular in women. Women with angina pectoris with low socio-economic status have a relative risk of 2.24 (95\% CI 1.17-3.26) compared with women with high socio-economic status. In men, no significant difference was found between the socio-economic status groups.
Analysing the referral rate of angina pectoris to the cardiologist showed that, in the low socio-economic status group, $53.3 \%$ of the men were referred compared to $21.3 \%$ of the women $(p=0.03)$.

\section{Course of inflammatory bowel disease}

The study population comprised 48 patients in the period 1985-2001: 22 women and 26 men (7). Mean age was 39.3 (SD 20.6) years in women and 39.7 (SD 16.9) years in men. Most patients suffered from one or more relapses in IBD. In men, relapse in IBD occurred almost 10 years after the time of diagnosis (first presentation) and in women after 6 years and 10 months (Figure 4). Also, the interval between the diagnosis of IBD and a symptom-free condition without drugs differed between men and women. Almost three-quarters of the male patients did not use any drugs after 151 months, while in women it was $58 \%$ after 204 months (Figure 5).

\section{Discussion}

We found that presentation to the general practitioner of more health problems by women was partly accounted for by disorders of the genitourinary system, but above all by screening and health education. This finding contradicts the assertions

Table I. Comparative incidence of health problems and their distribution (\%) for men and women, by severity.

\begin{tabular}{|c|c|c|c|c|c|}
\hline \multirow[b]{2}{*}{ Severity } & \multicolumn{3}{|c|}{ Incidence } & \multicolumn{2}{|c|}{ Distribution, \% } \\
\hline & Men & Women & Women minus men & Men & Women \\
\hline Serious & 46.4 & 51.8 & 5.4 & 2.9 & 2.3 \\
\hline Moderately serious & 333.0 & 423.2 & 90.0 & 20.9 & 18.6 \\
\hline Not serious & 937.3 & 1213.4 & 276.1 & 58.9 & 53.18 \\
\hline Not otherwise classified & 276. & 593.0 & 317.0 & 17.3 & 26.0 \\
\hline
\end{tabular}




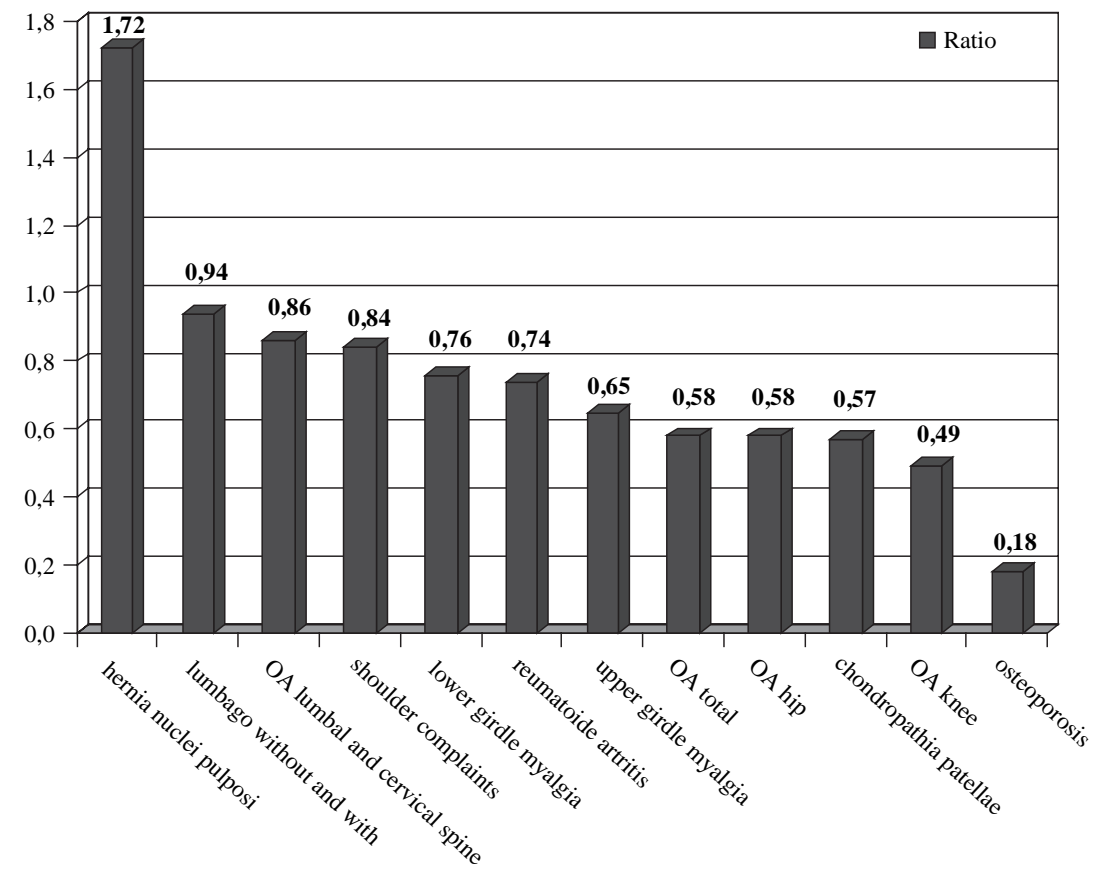

Figure 3. Male/female ratio of musculoskeletal disorders.

that nervous or vague complaints account for the greater number of female health complaints.

In accordance with the literature, we also found that health problems such as osteoarthritis of the knee and coronary heart disease varied according to socio-economic status. The lower the socioeconomic status, the higher was the morbidity (8). Surprisingly, in women with low socio-economic status, the relative risk of suffering from knee osteoarthritis or coronary heart disease is higher

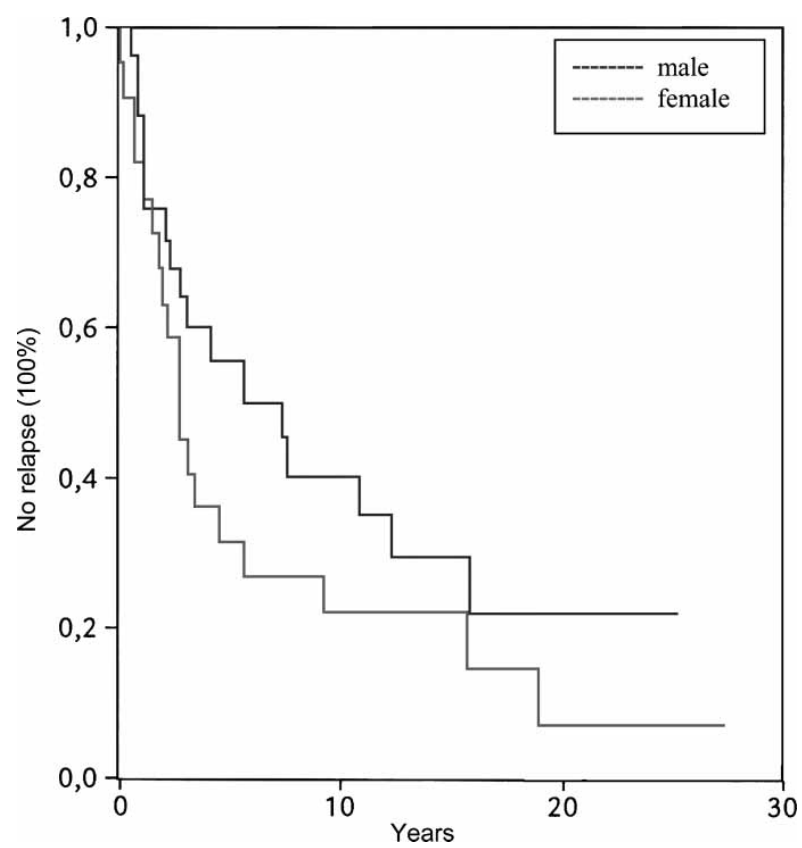

Figure 4. Time between diagnosis and first relapse in IBD. than for men with low socio-economic status. This outcome stresses the importance of paying attention to the concept of diversity, including socio-economic status, ethnicity, age, and gender as interrelated risk factors. Differences should not be considered as separate entities, since it seems clear that they do not operate independently but are strongly intertwined.

Another striking finding was that women with angina pectoris and low socio-economic status were

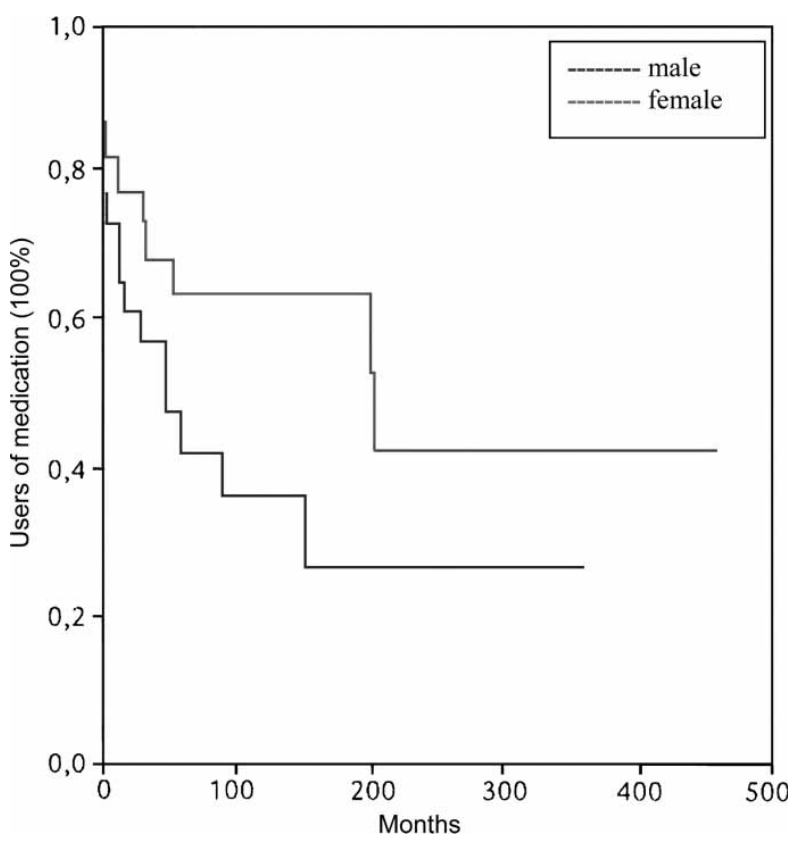

Figure 5. Time between diagnosis and start of medication in IBD. 
referred to cardiologists significantly less often than men. Women present more often with atypical angina pectoris, and a recent study confirmed that women have less access to invasive diagnostic and therapeutic procedures (9). Our figures revealed that low socio-economic status could strengthen the effect of undertreatment of women.

Finally, sex differences not only appear in the prevalence of health problems, risk factors, and access to secondary medical care, but also in the course of diseases. We studied inflammatory bowel diseases as just one example to illustrate that gender can make a difference in terms of a whole range of medical encounters, from first presentation to course and treatment $(7,10)$. A steadily increasing body of evidence has revealed disparities in symptoms and prognosis in many other health problems, such as HIV/AIDS, other sexually transmitted diseases, cardiovascular diseases, and autoimmune disorders (1). During the course of life, genetic information in the sex chromosome can be expressed differently between men and women, owing to a wide range of environmental factors. These genetic differences between the sexes also lead to differences in sensitivity and susceptibility to diseases between the sexes. Differences between the sexes can also be expressed non-uniformly in other biological phases of life, while certain factors, such as hormonal differences, can contribute to deviations in the development and course of diseases between men and women (11).

\section{References}

1. Lagro-Janssen ALM. Sex, gender and health. Developments in research. European Journal of Women's Studies 2007;14: 9-20.

2. Stoverinck MJM, Lagro-Janssen ALM, Weel C van. Sex differences in health problems. Diagnostic testing and referral in primary care. J Fam Pract 1996;43:567-76.

3. World Health Organization. WHO gender policy: integrating gender perspectives in the work of WHO. Geneva: World Health Organisation; 2002.

4. Gijsbers van Wiik CMT, Kolk AMM, Bosch WJHM van den, Hoogen $\mathrm{H}$ van den. Male and female morbidity in general practice: the nature of sex differences. Soc Sci Med 1992; 35:665-78.

5. Weel $\mathrm{C}$ van. The continuous morbidity registration Nijmegen. Background and history of a Dutch general practice database. (this issue).

6. Vogels EA, Lagro-Janssen ALM, Weel C van. Sex differences in cardiovascular disease: are women with low socioeconomic status at high risk. Br J Gen Pract 1999;49:963-6.

7. Kemenade $\mathrm{O}$ van, Roek M, Lagro-Janssen T. Chronische inflammatoire darmziekten in vier huisartspraktijken. [Inflammatory bowel diseases in four general practices.] Huisarts Wet 2004;47:615-9.

8. Chandola L. Social inequality in coronary heart disease: a comparison of occupational classifications. Soc Sci Med 1998;47:525-33.

9. Daly C, Clemens F, Lopez Sendon JL, Tavazzi L, Boersma E, Danchin N, et al. Gender differences in the management and clinical outcome of stable angina. Circulation 2006;113: 490-8.

10. Prickarts M, Lagro-Janssen T. Polymyalgia rheumatica in vier huisartspraktijken. [Polymyalgia rheumatica in four general practices]. Huisarts Wet 1999;42:597-601.

11. Wizemann TM, Pardue ML. Exploring the biological contribution to human health: does sex matter?. Washington, DC: National Academy Press; 2001. 